\title{
Modelling and Simulation of Complex Adaptive System: The Diffusion of Socio-Environmental Innovation in the RENDRUS Network
}

\author{
Aida Huerta Barrientos and \\ Yazmin Dillarza Andrade
}

Additional information is available at the end of the chapter

http://dx.doi.org/10.5772/67740

\begin{abstract}
Socio-environmental innovation is a process of social change that implies both the participation of agents on social and environmental initiatives and the generation and diffusion of relevant information, which lead social transformations for collective benefit. During the diffusion of socio-environmental innovations through a communication network, the information is created and shared among participants until mutual understanding is reached. In the case of National Network for Sustainable Rural Development (RENDRUS) network, getting innovations adopted is very difficult by people in rural communities due to the lack of effective communication channel. This study aims to develop a novel agent-based simulation model of socio-environmental innovation diffusion in the RENDRUS network based on complex adaptive systems approach. First, the conceptual model of socio-environmental innovation diffusion in the RENDRUS network based on complexity approach is developed. Then, an agent-based simulation model is implemented using Netlogo software, followed by the simulation model analysis and the design of plausible simulation scenarios. The simulation results illustrate how S-curve emerges from the interrelationships between agents considering endogenous and social cohesion effects. The conclusions argue that more social cohesion and popularity of socioenvironmental innovations between small rural producers and their organizations, governmental institutions, academic institutions and the knowledge society corresponds to less time to adopt socio-environmental innovations.
\end{abstract}

Keywords: complex adaptive systems, modelling and simulation, socio-environmental innovation, diffusion 


\section{Introduction}

As Sagarpa [1] explains, the Mexican National Network for Sustainable Rural Development (RENDRUS) network is a knowledge network for collaborative learning from producer to producer that contributes to diminishing the territorial inequality since it allows producers to know their own experiences. As Sagarpa [1] points out, the RENDRUS network contributes to food security through the development of capacities in rural communities, as well as the incorporation of new technologies that allow producers to establish areas for improvement in their productive, organizational and business processes. In this direction, the incorporation of innovations, the adoption of applied technology and the incorporation in the processes of extension to universities in rural areas led to the establishment of links between producers, their organizations and the knowledge society to generate sustainable rural development in Mexico.

On the one hand, according to Quiroga and Barrera Gaytan [2], the socio-environmental innovation is a process of gradual change through action research in localized territories, which implies that a set of actors, based on their own interests, mission and capacity, participate in specific activities (scientific, technological, environmental, cultural, organizational, financial and commercial) whose orientation is not only to give a creative answer to linked problems of rural development and conservation of natural resources but also to generate learning that lead to the autonomy of the actors and structural transformations that are reflected in the collective benefit. Following Ref. [2], the socio-environmental innovation seeks to generate a flow of relevant information through channels and networks of interaction, promote the process of generation and diffusion of innovations and emphasize as central aspect the interconnection of these channels and networks. On the other hand, as Rogers [3] states, the diffusion of innovation is a communication process through certain channels over time among the members of a social system, where participants create and share information with one another in order to reach a mutual understanding. As described by Rogers [3], from the late 1920s to the early 1980s, the nine major diffusion traditions were anthropology, early sociology, rural sociology, education, medical sociology, communication, marketing, geography and general sociology; the problem faced was the research designs that consisted mainly of correlational analysis on data gathered in one-shot surveys of respondents (adopters and/or potential adopters of an innovation). More recently, the complex adaptive system (CAS) approach was used to analyse the spread of an innovation through a complex social system. The concept of complex adaptive system was introduced in 1967 by Walter Buckely to define a system in which large networks of essential components, without central control and simple rules of interaction between the network components, give rise to complex collective behaviour, sophisticated information processing and adaptation via learning and evolution. Derived from the interactions between CAS and the environment, the internal state of CAS changes through time-forming trajectories that may or may not converge to certain regions of its state space such as the attractors and repulsors, depending on the function they perform. In the study of CAS, it is interesting to know its emergent properties, those that arise at higher structural levels and are due to the interactions between the elements at a lower structural level. The approach of modelling and simulation has been used to better understand 
the dynamic and evolution of CAS. As suggested by Viale and Pozzali [4], complex adaptive system research can teach us a series of useful lessons, especially those features necessitating the consideration of innovation systems as a complex adaptive system because studying the dynamics of innovation is a complex task.

As Yilmaz [5] points out, from the complexity perspective, the innovation is conceptualized as a CAS phenomenon that occurs at different scales, including individual, group and organizational levels. For instance, at the collective level, innovation can be conceptualized as global property that emerges from the local interactions of actors within a CAS, which influence one another in response to the influence they receive. In this direction, Yilmaz [5] suggests that in a study of CAS it is necessary to know structural and behavioural conditions, for the emergence and sustainment of innovation. As reported by Yilmaz [5], the benefits of abstracting a conceptual model of collective innovation based on the CAS perspective are as follows:

- The empirical understanding of innovation is improved through a formal agent-based simulation model testbed by making it possible to explore particular types of observed regularities via simulation scenarios.

- Agent-based simulation models can be used as computational laboratories for the discovery of organizational designs that are conducive to innovation.

- Agent-based simulation acts as theory generation enabler by facilitating a better understanding of innovation complex dynamics over a full range of feasible configurations and behaviour of complex social systems.

In the study of diffusion of innovations based on CAS perspective, Rogers et al. [6] explores the actual and potential hybridization of these two system theories, relying on illustrations from historical to practical applications of the diffusion of innovation model credited by Rogers [3], particularly the STOP AIDS communication campaign in San Francisco, USA. Modelling the diffusion of environmental innovations not only helps understanding diffusion processes but also enables researchers to develop scenarios of future use of these innovations, therefore indicating possible ways towards a more sustainable future, as Schwarz [7] stated. In the case study of the RENDRUS network, there is much interest in the diffusion of socio-environmental innovations in territories because it is one way to more sustainable use of natural resources. This chapter presents a novel agent-based simulation model of the diffusion of socio-environmental innovation in the RENDRUS network based on the CAS perspective in order to understand the diffusion process in the network for achieving sustainable rural development. The study is of relevance because obtaining an innovation and best practices in the value chain adopted is often very difficult to people in territories. Additionally, we consider that CAS approach gives a better basis for understanding the diffusion of socio-environmental innovation as an evolutionary network of functional elements that interact exhibiting characteristics of a complex adaptive system. An important aspect of the resulting simulation model is that it provides an analytical tool to support the decision-making of governmental institutions towards sustainable rural development in Mexico. 
The chapter is divided into five main sections. In Section 2, the conceptual for diffusion of socio-environmental innovation in the RENDRUS network based on CAS approach is developed. In Section 3, the agent-based simulation model for diffusion of socio-environmental innovation in the RENDRUS network is implemented using Netlogo software. The agentbased simulation model analysis and the design of plausible simulation scenarios for achieving sustainable rural development in Mexico are presented in Section 4. The concluding remarks are drawn in Section 5.

\section{A conceptual model for diffusion of socio-environmental innovation in the RENDRUS network based on CAS perspective}

\subsection{Conceptual model development}

According to Sayama [8], the various modelling approaches can be put into the following major families:

- Descriptive modelling. In this approach, modellers try to specify the state of a system at macro-level at a given time point, capturing what the system looks like. This can be done taking a picture, creating prototypes such as physical models or using quantitative methods such as statistic models.

- Rule-based modelling. In this approach, modellers try to come up with dynamical rules at micro-level that explain the observed macro-behaviour of a system. The modelling methodologies mainly used are cellular automata, network models, agent-based models and dynamical equations.

\subsection{Agent-based modelling and simulation (ABMS)}

CAS perspective concerns with elements called agents that learn or adapt in response to rulebased non-linear interactions with other agents generating the behaviour and the hierarchical structure of a CAS; particular combinations of agents at one level become agents at the next level up [9]. As the result of the non-linear interactions, the agents are able to cooperate and evolve, improving in certain cases their fitness over time [10]. In the mid-1990s, the ABMS computational approach was recognized by Holland as a foundational methodology to the study of CAS [11]. ABMS is a form of computational modelling whereby a phenomenon is modelled in terms of agents and their interactions [12] using the bottom-up perspective in the sense that the system behaviour that we observe in the model emerges from the bottom of the system by the direct interrelations of the agents from the basis of the model [13]. So, it is possible to understand the simple interaction rules between agents at local level. The definition of a space scale is fundamental in ABMS, while the time scale varies in discrete steps. From the computational perspective, the software that is used to program agents has its origins in the areas of Artificial Intelligence, especially in the subfield of Distributed Artificial Intelligence [14, 15], whose objective is the study of agent's properties and the design of networks of interaction 
between them. It was suggested by Wooldridge and Jennings [16] that computational agents are typically characterized as follows:

- Autonomy: the agents had direct control of their actions and their internal state.

- Social skills: the agents interacted with other agents through a computational language.

- Reaction: agents were able to perceive their environment and respond to it. The environment could be the physical world, a virtual world or a simulated world that includes other agents.

- Proactivity: because the agents reacted to their environment, they themselves had to take the goal-oriented initiative.

In general, the environment of agents is interpreted in terms of a metaphorical vocabulary of beliefs, desires, motives and emotions, which are generally applied more in the description of people. The agent's attributes typically modelled are knowledge and beliefs, inferences, social models, representation of knowledge, goals, planning, language and emotions. According to Macal and North [17], an agent-based model contains the following four elements (see Figure 1):

- Agents, their attributes and environment.

- Relationships between agents and the rules of interaction.

- A connectivity network that defines how and with whom agents interact.

- Agent's environment that interacts with agents, interchanging information.

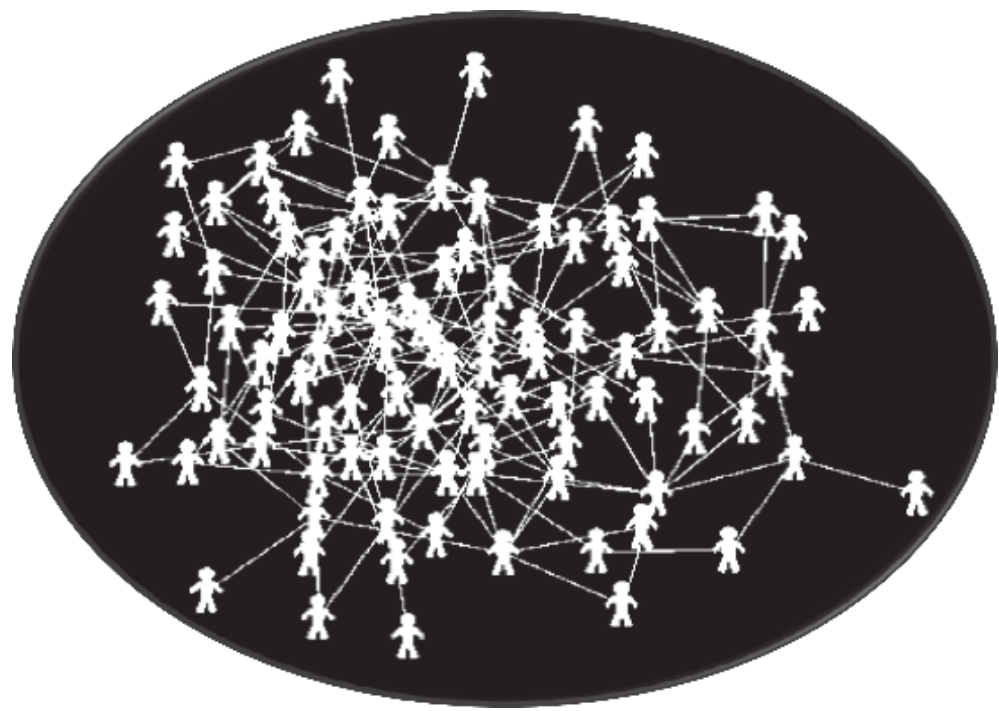

Figure 1. A network of elements in a typical agent-based model. 


\subsection{The RENDRUS network modelled as a CAS}

The RENDRUS network conceptualized as a CAS presents the following characteristics (see Figure 2):

- Multiple key heterogeneous agents. Small rural producers and their organizations, governmental institutions, academic institutions and the knowledge society.

- Different structural levels. At micro-level, the RENDRUS network is constituted by rural producers interacting with one another, and with governmental and academic institutions, while at macro-level, the collaborative learning emerges for leading to the autonomy of rural producers and the structural transformations, which are reflected in the collective benefit.

- Intrinsic diversity among its key agents. Small producers from the 32 Mexican federal states are product of the exposure of their own experiences within a socio-economic, cultural and environmental context. So, the incorporation and adoption of new socio-environmental innovations, in order to establish areas for improvement in productive, organizational and business processes, are determined by local requirements.

- Functional dynamics. The RENDRUS network is an open system that exchanges relevant information containing producer's experiences, with the complex environment. In order to survive (increasing the participation of rural producers and governmental and academic institutions), the RENDRUS network has to adapt itself to new conditions imposed by the environment, adjusting its functional units through the modification and selection of new socio-environmental innovations.

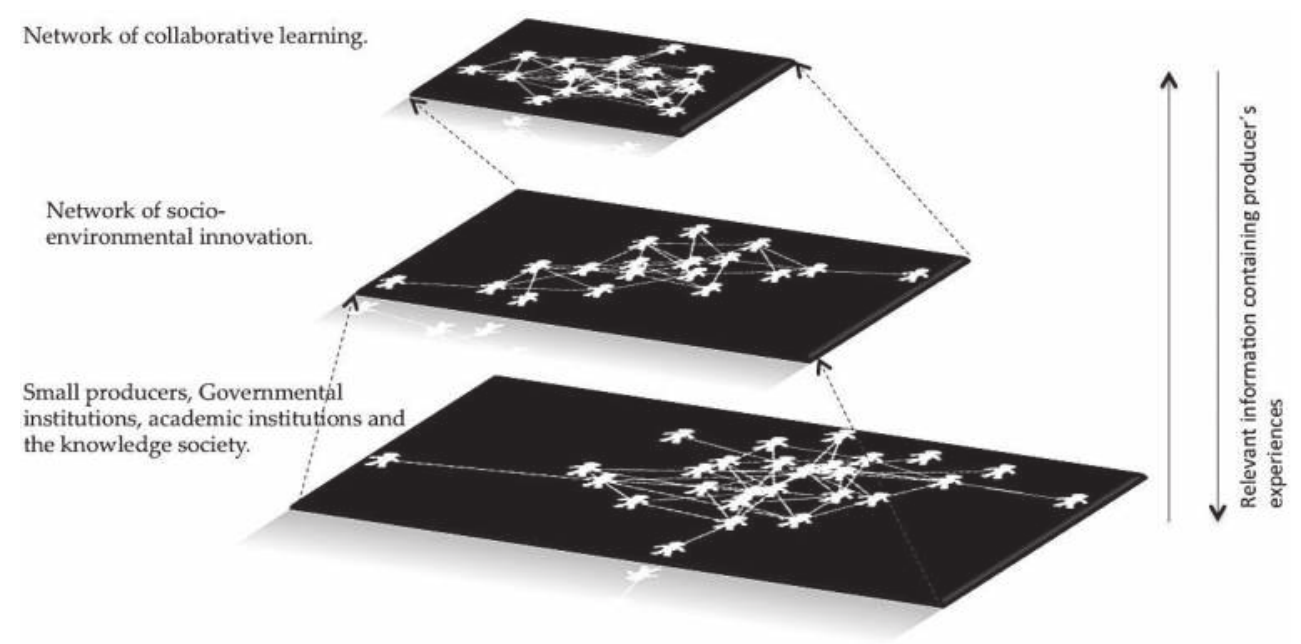

Figure 2. The conceptual model of the RENDRUS network based on CAS approach. 
- The impact of the social structure. The interactions between members of the RENDRUS network are not random but are rather limited by social networks, both internally and externally. The social structure and socio-cultural interactions between members have a crucial effect on the process of evolution of the adoption of socio-environmental innovations. In this case, the principle of the social structure is based on the community.

\subsection{The diffusion of socio-environmental-innovation model in the RENDRUS network based on CAS approach}

The diffusion in the RENDRUS network is the process by which socio-environmental innovation is communicated through certain channels (meetings, Internet, etc.) over time among rural producers and their organizations, governmental institutions and academic institutions. In this case, when new answers to complex problems regarding sustainable rural development emerge from the interactions between rural producers and governmental and academic institutions and these are diffused and adopted or rejected by rural producers, some alterations occur at functional and structural level of the RENDRUS network, originating a social change due to the activation of peer networks about such socio-environmental innovation in the next four areas: transformation of primary, agricultural and livestock production, rural industry and marketing, rural non-agricultural services and handicrafts, and rural extension.

Following Ref. [3], we present the five attributes of socio-environmental innovations of the RENDRUS network as follows:

- Relative advantage. The degree to which rural producers perceive the socio-environmental innovation as being better than the practice it supersedes. The exchange of innovationevaluation information between rural producers of the RENDRUS network, using the communication network, lies the diffusion process.

- Compatibility. The degree to which the socio-environmental innovation is perceived by rural producers as consistent with the existing socio-cultural values, past experiences and specific needs of potential adopters.

- Complexity. The degree to which rural producers perceive the socio-environmental innovation as relatively difficult to understand and use.

- Trialability. The degree to which the socio-environmental innovation may be experimented with on a limited basis.

- Observability. The degree to which the results of the socio-environmental innovation are visible to other rural producers.

In summary, socio-environmental innovations of the RENDRUS network that are perceived as relatively advantageous, compatible with existing socio-cultural values, beliefs and experiences, relatively easy to adapt, observable and divisible for trial, will be adopted more rapidly by rural producers.

As discussed by Bass [18], in the literature, two models for how innovations diffuse through social systems are recognized: endogenous and exogenous. On the one hand, in the endogenous 
diffusion model, how fast an innovation spreads is a function of its own popularity between the members of the social system. Following Ref. [18], in this case the proportion of the social system that has adopted the innovation over time starts out slow, slowly builds to a critical mass, where it achieves exponential growth, and finally levels off as it saturates the social system. The model, as seen in Figure 3, is a bell-shaped curve that shows the data about the general endogenous diffusion process on a frequency basis, whereas the s-shaped curve shows the same data on a cumulative basis [3]. On the other hand, in the exogenous diffusion model, potential adopters respond directly to exogenous effects.

From the CAS perspective, both variety and reactivity are necessary for the diffusion of an innovation [6]. Variety refers to the diverse population for emergence and adaptation and it is necessary for information exchange that takes place between an innovation sender and an innovation receiver, whereas reactivity refers to the sensitivity to change where only those populations with adaptability to change can survive at the higher fitness thresholds that occur during cascading mutation/extinction [6]. The information exchange between an innovation sender and an innovation receiver is a critical process in the diffusion of socio-environmental innovation in the RENDRUS network. It means that more information about best practices and new technology corresponds to less uncertainty on rural producer perceptions about relative advantage, compatibility and complexity of innovations, creating better conditions to the emergence and adaptation. The diffusion of socio-environmental-innovation model in the RENDRUS network based on CAS approach contains the following four elements (see Figure 4):

- Heterogeneous agents. Small rural producers and their organizations, governmental institutions, academic institutions and the knowledge society. The agents ( $a g)$ have two attributes: the number of neighbours having already adopted the socio-environmental innovations (an) and a random threshold for adoption $(r t)$ with a normal distribution $N(100,25)$ to satisfy the heterogeneity condition. The Bass model [18] allows evaluating the evolution of both endogenous and exogenous diffusion as follows:

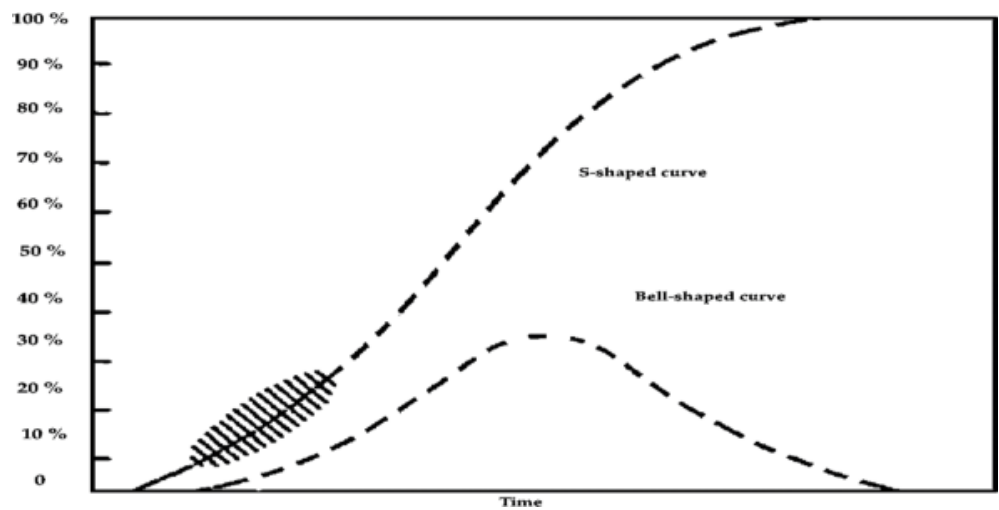

Figure 3. The endogenous diffusion model of an innovation over time by members of a social system, adopted from Ref. [3]. 


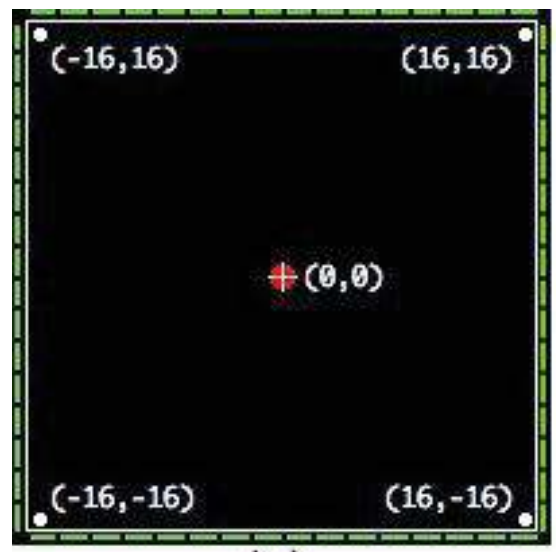

(a)

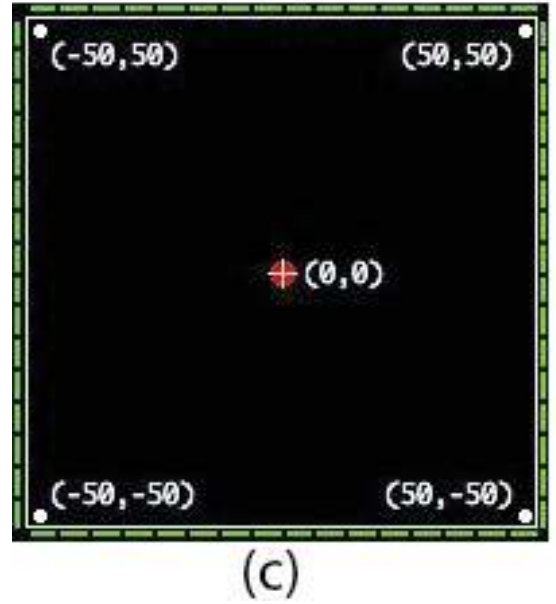

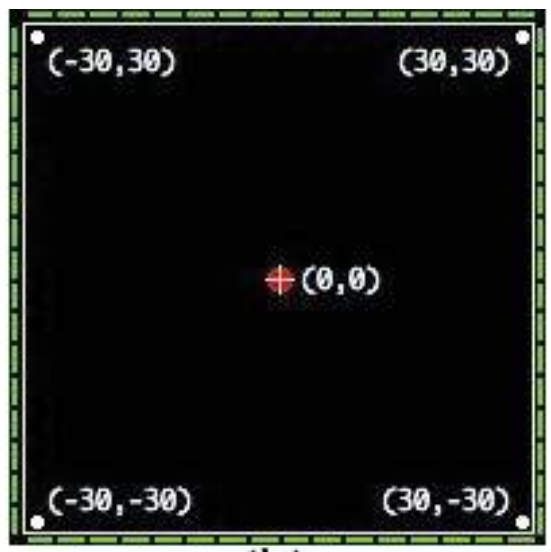

(b)

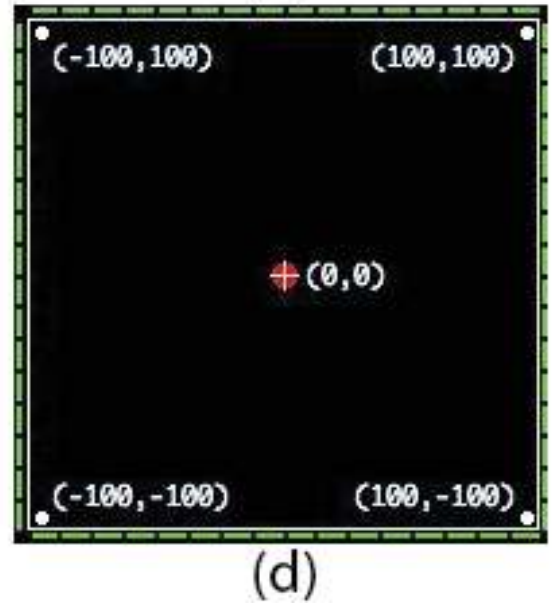

Figure 4. The Netlogo grid based on four different values for MAX-PXCOR and MAXPYCOR, (a) MAX-PXCOR = MAXPYCOR $=16$ with a total of 256 patches, (b) MAX-PXCOR $=$ MAXPYCOR $=30$ with a total of 900 patches, (c) MAX$\mathrm{PXCOR}=\mathrm{MAXPYCOR}=50$ with a total of 2500 and $(\mathrm{d}) \mathrm{MAX}-\mathrm{PXCOR}=\mathrm{MAXPYCOR}=100$ with a total of 10,000 patches .

$$
f_{t}=\left(p+q F_{t}\right)\left(1-F_{t}\right),
$$

where $F_{t}$ describes the proportion of potential adopters having already adopted the innovation, $p$ coefficient refers to the exogenous effect, and $q F_{t}$ coefficient indicates the endogenous effect at time $t$. The endogenous diffusion model is a special case of Eq. (1) when $p=0$. Additionally, one mechanism assumed by endogenous diffusion models is contagion, whereby those who have adopted the innovation directly promote the innovation to those with whom they are in contact [19]. In this study, we use a variant of endogenous diffusion model based on threshold called information cascade, whereby the number of prior 
adoptions is a source of credible information. The importance of threshold models is the aggregation of popularity [20].

- Rules of interaction. Agents interact with their neighbours and the decision for adopting socio-environmental innovations depends if $r t$ value is less than a non-linear function evaluated in terms of the number of adopters $(n a)$, the endogenous effect (ee) and the social cohesion between agents $(s c)$ whose range can be dynamically updated to revise the $r t$ of an agent, as follows:

$$
r t<e e^{*}\left(\frac{n a}{a g}\right)+\left(a n^{*} s c\right)
$$

- A connectivity network. The network is built generating each agent from 0 to 4500 . Once an agent is created (old agent), a new referred position is searched for five neighbours, to create the new agent.

- Agents interchange information indicating the number of their own neighbours having already adopted the socio-environmental innovations (prior adoptions).

\section{Agent-based simulation model for the diffusion of socio- environmental innovation in the RENDRUS network}

\subsection{Netlogo simulation software}

Netlogo software, first developed in the late 1990s by Uri Wilensky, is a general-purpose agent-based modelling language used worldwide that provides a graphical modelling environment [21], freely available on the Netlogo website (https://ccl.northwestern.edu/netlogo). As Wilensky [22] explains, it is an extension of the Logo language in which user controls a graphical turtle by issuing commands, and it includes a grid of patches, each patch is a cell computationally active. Turtles and patches are self-contained objects with internal local state. In Netlogo models, time passes in discrete steps, called ticks. Netlogo allows to scale space and time, such as $1 \mathrm{~m}^{2}=1$ patch and one tick can represent a minute or a day, and so on. The grid can be adjusted using the MAX-PXCOR (horizontal direction) and MAXPYCOR (vertical direction) in two-dimension (2D), to create a larger or shorter world keeping the view a manageable size on the main screen, for a total of MAX-PXCOR * MAXPYCOR patches. Figure 4 illustrates the Netlogo grid with different MAX-PXCOR and MAXPYCOR values, where the worlds wrap both horizontally and vertically. As seen in Figure 5, the grid can be visualized in $2 \mathrm{D}$ and three dimension (3D).

\subsection{The agent-based simulation model implementation}

Our agent-based simulation model implemented in Netlogo software produces four artificial worlds, each populated by 1000, 2000, 3000 and 4500 heterogeneous agents, respectively. In order to ensure that the simulation parameters are realistic, we base our agents upon RENDRUS network data about small rural producers available on the RENDRUS website 


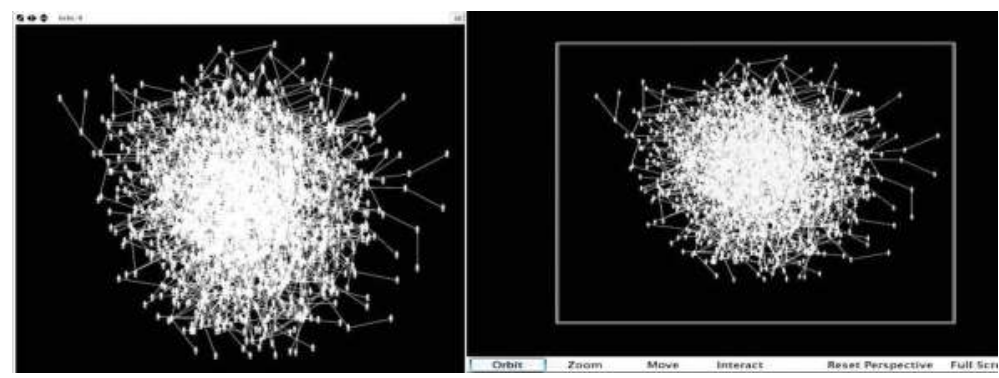

Figure 5. The Netlogo grid including turtles and patches in 2D and 3D. Netlogo grid space of patches in (a) 2D and (b) 3D.

(http://rendrus.extensionismo.mx/rendrus/rendrus). The artificial worlds created vary in the following experimental factors: (a) endogenous and (b) social cohesion effects. We use them to study the rate of adoption, as the relative speed with which socio-environmental innovations are adopted along time. The endogenous effects refer to the popularity of socioenvironmental innovations, based on the relative advantage that rural producers perceive from the socio-environmental innovation as being better than the practice it supersedes. The popularity increases when the results of the socio-environmental innovation are visible to other rural producers, whereas social cohesion effects represent the compatibility perceived by rural producers about the innovation as consistent with the existing socio-cultural values, past experiences and specific needs of potential adopters. Table 1 shows the endogenous and social cohesion effect's numerical values used in the simulation model.

The time variable allows us to plot the number of RENDRUS members adopting the socioenvironmental innovation along time. In this model, each 'tick' from Netlogo represents 1 day in the time scale. The simulation model starts with one agent who has already adopted the socioenvironmental innovation. The simulation model ends after 1200 simulation days (ticks). As can be seen in Figures 6-9, the proportion of adopters starts out slow due to the interactions between heterogeneous agents then, slowly builds to a critical mass growing exponentially, and finally the number of adopters saturates the artificial world that represents the RENDRUS network.

\subsection{Agent-based simulation model validation}

As Wilensky and Rand [12] explains, simulation model validation is the process of determining whether the implemented simulation model corresponds to some phenomenon in

\begin{tabular}{lll}
\hline Simulation parameter & Lower value & Upper value \\
\hline Endogenous effects $(e e)$ & 10 & 20 \\
Social cohesion effects $(s c)$ & 30 & 50 \\
\hline
\end{tabular}

Table 1. The range of simulation parameters. 

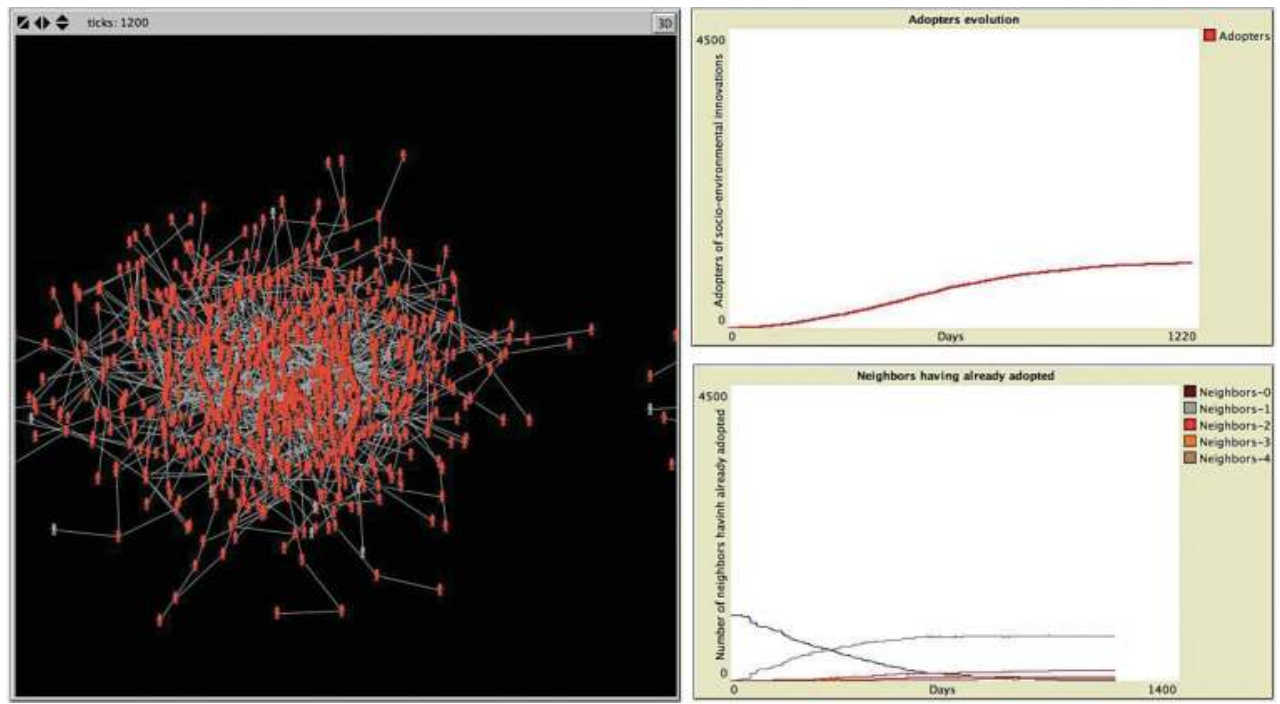

Figure 6. An artificial world populated by 1000 heterogeneous agents, $e e=10, s c=30$.
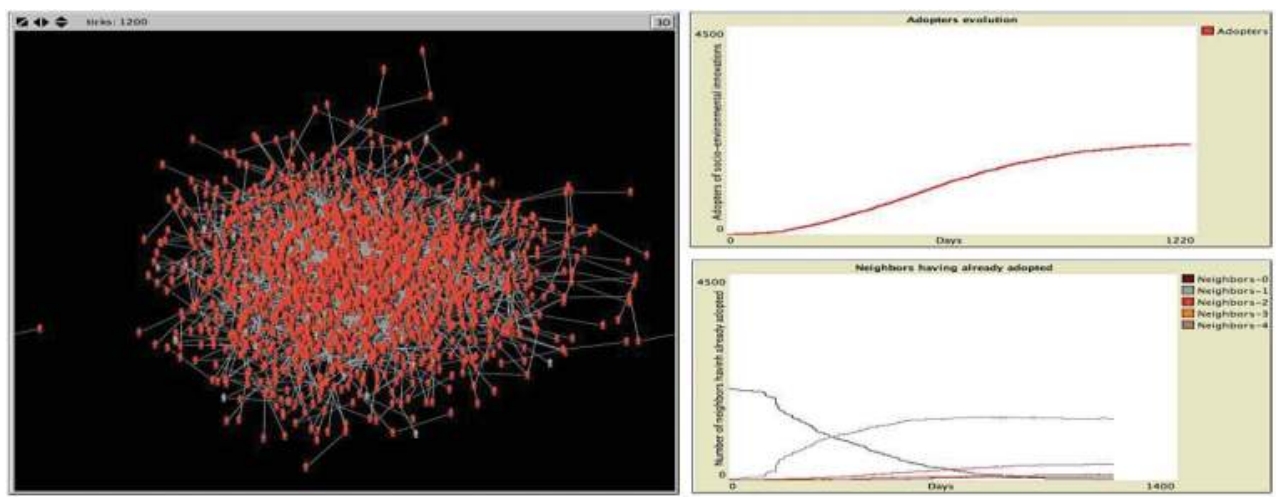

Figure 7. An artificial world populated by 2000 heterogeneous agents, $e e=10, s c=30$.

the real world. Our agent-based simulation model was validated using the dynamic technique called sensitivity analysis. Through it, values of simulation parameters are systematically changed over some range of interest and the simulation model's behaviour is observed [23]. This technique allows identifying the simulation parameters to which the simulation model behaviour is very sensitive. The simulation parameter considered to carry out the sensitivity analysis is the social cohesion effect $(s c)$. In this case, the agent-based simulation model is executed considering two values for this parameter: 20 and 50. The artificial world considers 4500 heterogeneous agents. The endogenous effect (ee) is fixed on 10. The simulation model ends after 1200 simulation days (ticks). The simulation results are illustrated 

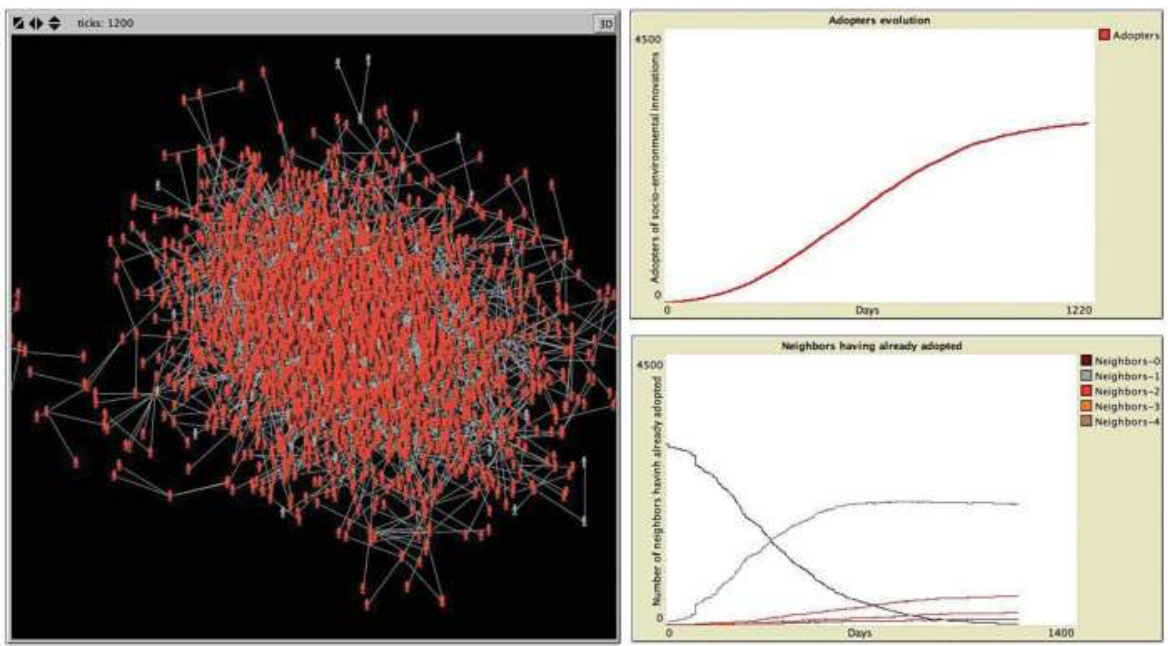

Figure 8. An artificial world populated by 3000 heterogeneous agents, $e e=10, s c=30$.
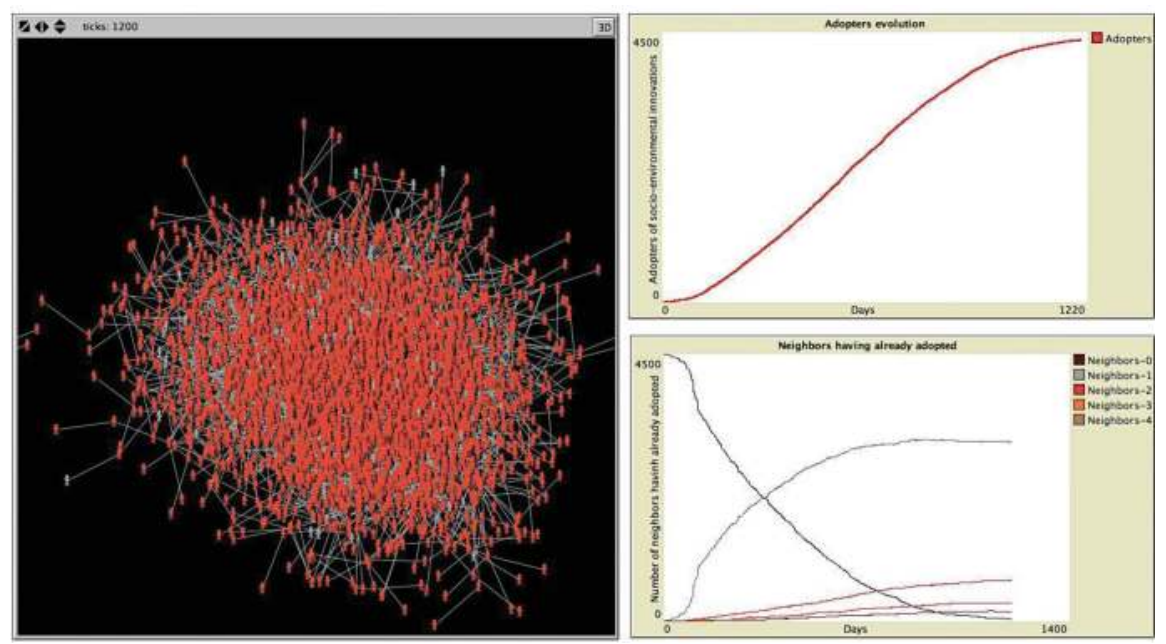

Figure 9. An artificial world populated by 4500 heterogeneous agents, $e e=10, s c=30$.

in Figures 10 and 11. As can be seen in Figure 10, the proportion of adopters reaches near $50 \%$ of the total population in 1200 ticks. In this case, the rate of adoption, as the relative speed with which socio-environmental innovations are adopted along time, is very slow. On the contrary, as can be seen in Figure $\mathbf{1 1}$ the proportion of adopters starts out slow then, grows exponentially, and finally just in 298 ticks the number of adopters saturates the artificial world. Therefore, more social cohesion among agents in the RENDRUS network corresponds to less time to adopt socio-environmental innovations and vice versa, and less 

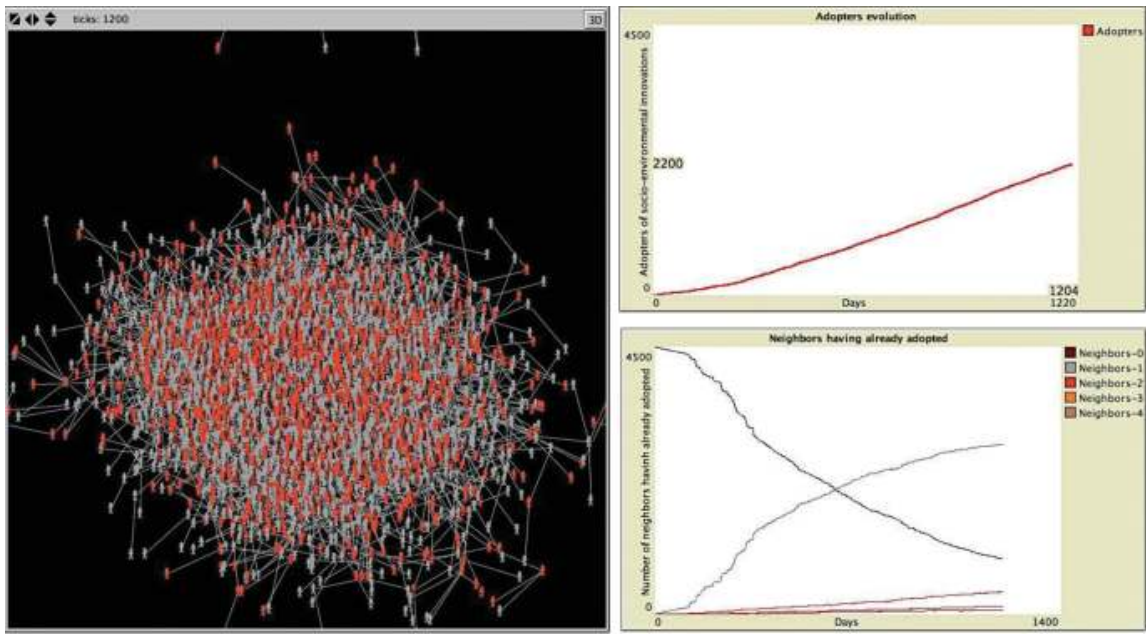

Figure 10. An artificial world populated by 4500 heterogeneous agents, $e e=30, s c=20$.
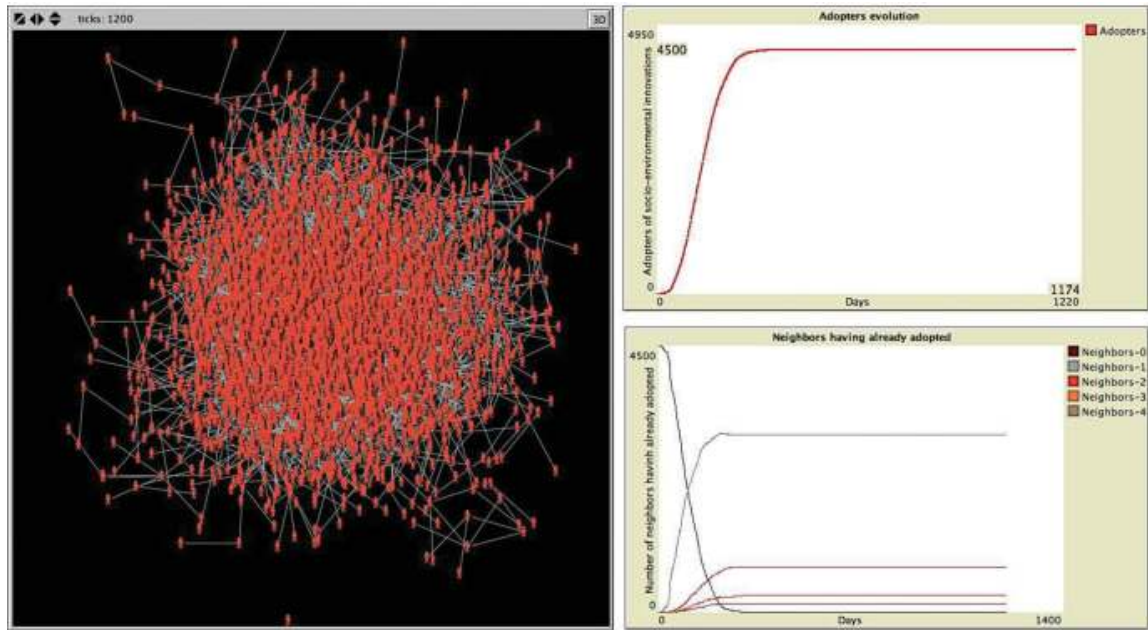

Figure 11. An artificial world populated by 4500 heterogeneous agents, $e e=10, s c=50$.

social cohesion corresponds to much more time to the adoption. In this case, the innovation adoption is very sensitive to the social cohesion of the RENDRUS network.

\subsection{Agent-based simulation model verification}

As Wilensky and Rand [12] explains: simulation model verification is the process of making sure that the simulation model has been correctly implemented on a computer using simulation software. In Netlogo software, the compilation and execution processes that prepare the model to run happen behind the scenes and require no intervention by the user; however, syntax and 
other coding errors that cause runtime errors during compilation and execution process interrupt the simulation [24]. In this phase, we eliminated the 'bugs' from the code so the model was correctly implemented, free of errors.

\section{Agent-based simulation model analysis}

\subsection{Designing plausible simulation scenarios for achieving sustainable rural development in Mexico}

Sustainable development is a process of change in which the exploitation of resources, the direction of investment, the orientation of technological development and institutional change are made consistent with both future and present needs [25]. Mexico is a country committed to addressing sustainable development, as demonstrated by the actions undertaken over the last few years [26]. For instance, in 2001 the Mexican Congress approved the Law on Sustainable Rural Development, on which the sustainable rural development is defined as the improvement of social welfare and economic activities in the territory specified outside the urban centres considered in accordance with the applicable provisions, ensuring the permanent conservation of natural resources, biodiversity and ecosystem services in that territory. Another tangible result is the National Network for Sustainable Rural Development (RENDRUS) that promotes a series of annual meetings for exchanging and evaluating successful experiences between rural producers, seeking a process of collective learning at different levels. In this context, we consider the design of plausible simulation scenarios essential for a better diffusion of socio-environmental innovations through the RENDRUS network in order to improve the social welfare in rural areas, ensuring the permanent conservation of natural resources, biodiversity and ecosystem in such areas.

Scenarios were introduced over 50 years ago firstly by Herbert Kahn as a means to overcome the limits of reductionist thinking in response to the difficulty of creating accurate forecasts. In scenarios, the notion of wholly predictable futures of a system is rejected and instead the alternative futures that explore the paths to each, emphasizing the need to attend to disruptive change as normal, are seen [27]. Plausibility-based scenarios described by Schoemaker [28] are useful approaches in situations characterized by increasing uncertainty and complexity. According to Peterson et al. [29], in order to build scenarios, it is important to specify what is known and unknown about the system's dynamics then, the alternative ways that the system could evolve must be identified. After that, a set of scenarios is built, through which our current thinking about the system should be expanded. Following Ref. [29], the dynamics of scenarios must be plausible; neither nature nor the agents involved in the scenario should behave in implausible ways. The most important part of a scenario's plausibility is likely to be the behaviour of agents. In this direction, the behaviour of small rural producers and their organizations, governmental institutions, academic institutions and the knowledge society could improve the diffusion of socio-environmental innovations through the RENDRUS network. Our simulation model considers that agent's behaviour is influenced by the $r$ t parameter (the random threshold for adoption). The $r t$ indicates the popularity of the socio-environmental innovations. Table 2 shows the range of simulation parameters for plausible simulation scenarios. 


\begin{tabular}{lll}
\hline Simulation parameter & Lower value & Upper value \\
\hline Endogenous effects $(e e)$ & 10 & 10 \\
Social cohesion effects $(s c)$ & 50 & 50 \\
Random threshold for adoption $(r t)$ & $N(50,15)$ & $N(70,15)$ \\
\hline
\end{tabular}

Table 2. The range of simulation parameters for plausible simulation scenarios.

\subsection{Analysis of plausible scenarios}

Figure 12 illustrates the evolution of adopters over time. We observe that the proportion of adopters starts out fast forward then, grows exponentially and finally just in 15.6 days (ticks) saturates the artificial world.

As seen in Figure 13, the proportion of adopters starts out slow then, grows exponentially and finally just in 88.5 days (ticks) saturates the artificial world. Therefore, more popularity of socio-environmental innovations $(r t)$ among small rural producers and their organizations, governmental institutions, academic institutions and the knowledge society corresponds to less time to adopt it by the RENDRUS network considered as a whole.
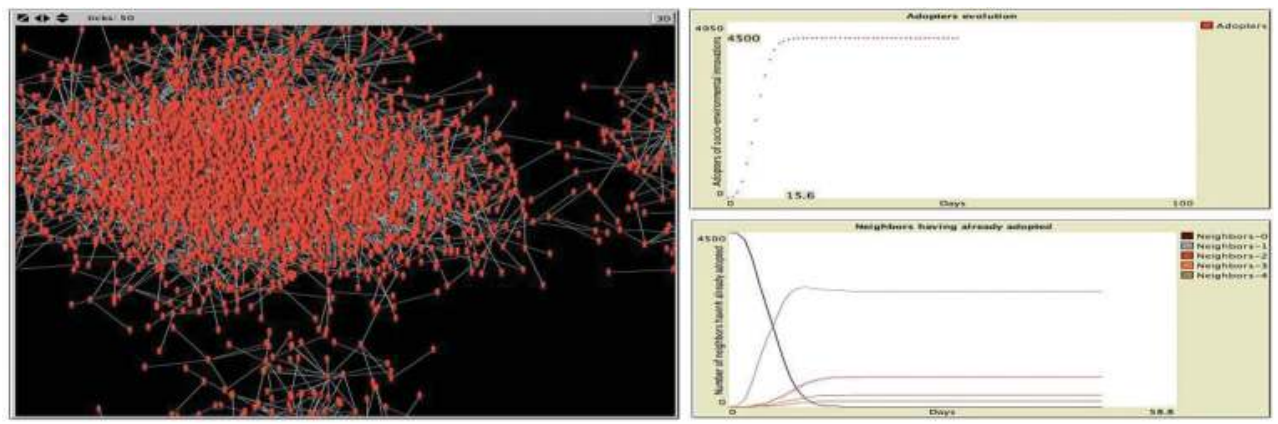

Figure 12. Artificial world populated by 4500 heterogeneous agents, $e e=10, s c=50, r t \sim N(50,15)$.
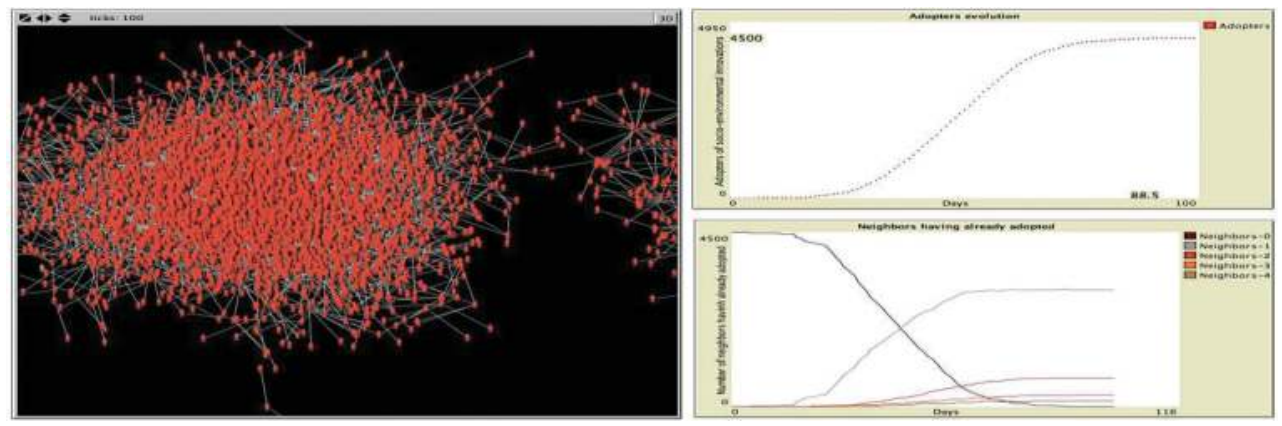

Figure 13. Artificial world populated by 4500 heterogeneous agents, $e e=10, s c=50, r t \sim N(70,15)$. 


\section{Concluding remarks}

The socio-environmental innovation is defined as a process of gradual social change through action research in localized territories, whose orientation is not only to give a creative answer to linked problems of rural development but also to generate learning that lead to the autonomy of the actors that is reflected in the collective benefit. The RENDRUS network is a governmental initiative to establish links between producers, their organizations and the knowledge society to generate sustainable rural development in Mexico. This chapter presented a novel agent-based simulation model of the diffusion of socio-environmental innovation in the RENDRUS network based on the CAS perspective in order to understand the diffusion process in the network. Modelling and simulating the socio-environmental innovation diffusion is important because it helps us understand the use of innovations that can support the sustainable rural development. From the simulation results, we observed on the one hand that more social cohesion among small rural producers and their organizations, governmental institutions, academic institutions and the knowledge society corresponds to less time to adopt socio-environmental innovations, and on the other hand that more popularity of socio-environmental innovations among them corresponds to also less time to adopt it. In conclusion, the diffusion of socio-environmental innovation to contribute to the sustainable rural development in Mexico depends upon a deep understanding of the interactions among rural producers and their organizations, governmental and academic institutions.

\section{Author details}

Aida Huerta Barrientos ${ }^{1,2 *}$ and Yazmin Dillarza Andrade ${ }^{1}$

*Address all correspondence to: aida.huerta@comunidad.unam.mx

1 Faculty of Engineering, National Autonomous University of Mexico, Mexico City, Mexico

2 The Complexity Sciences Center, National Autonomous University of Mexico, Mexico City, Mexico

\section{References}

[1] Sagarpa [Internet]. 2016. Available from: http://rendrus.extensionismo.mx/rendrus/

[2] Quiroga C A, Barrera Gaytan J F. Evaluar la innovación socioambiental? In: Bello Baltazar E, Naranjo Piñera E J, Vandame R, editors. La otra innovación para el ambiente y la sociedad en la frontera sur de México. Mexico: El Colegio de la Frontera Sur; 2012.

[3] Rogers, E M. Diffusion of innovations. London: Collier Macmillan Publishers; 1983.

[4] Viale R, Pozzali A. Complex adaptive systems and the evolutionary triple helix. Critical Sociology. 2010; 36 (4). 
[5] Yilmaz L. Innovation systems are self-organizing complex adaptive systems. Association for the Advancement of Artificial Intelligence. 2008.

[6] Rogers E M, Medina U E, Rivera M A, Wiley C J. Complex adaptive systems and the diffusion of innovations. The Public Sector Innovation Journal. 2005; 10 (3): 2-26.

[7] Schwarz N. Agent-based modeling of the diffusion of environmental innovations. An empirical approach. In: Proceedings of the 5th International EMAEE Conference on Innovation; 17-19 May 2007.

[8] Sayama H. Introduction to the modeling and analysis of complex systems. New York: Open SUNY Textbooks; 2015.

[9] Holland J H. Complexity, a very short introduction. New York: Oxford University Press; 2014.

[10] Huerta-Barrientos A, Flores de la Mota I. Modeling sustainable supply chain management as a complex adaptive system: the emergence of cooperation. In: Krmac E, editor. Sustainable supply chain management. Croatia: InTech; 2016. DOI: 10.5772/62534

[11] Holland J H. Hidden order: how adaptation builds complexity. USA: Perseus Books; 1995.

[12] Wilensky U, Rand W. An introduction to agent-based modeling. Cambridge: The MIT Press; 2015.

[13] Miller J H, Page S E. Complex adaptive systems. An introduction to computational models of social life. Princeton: Princeton University Press; 2007.

[14] Bond A H, Gasser L. Readings in distributed artificial intelligence. Los Altos, CA, USA: Morgan Kaufmann; 1988.

[15] Chaib-draa B, Moulin B, Mandiau R, Millot P. Trends in distributed artificial intelligence. Artificial Intelligence Review. 1992; 6: 35-66.

[16] Wooldridge $M$, Jennings $N$ R. Intelligent agents: theory and practice, Knowledge Engineering Review. 1995; 10: 115-152.

[17] Macal C, North M. Introductory tutorial: agent-based modeling and simulation. In: Proceedings of the Winter Simulation Conference, December 2011. p. 1456-1468.

[18] Bass F M. A new product growth for model consumer durables. Management Science. 1969; 15: 215-227.

[19] Rossman G. The diffusion of the legitimate and the diffusion of legitimacy. Sociological Science. 2014; 1: 49-69.

[20] Granovetter M. S. Threshold models of collective behavior. American Journal of Sociology. 1978; 83: 1420-1443.

[21] Dorner D. The logic of failure: recognizing and avoiding error in complex situations. New York: Basic; 1997. 
[22] Wilensky U. GasLab: An extensible modeling toolkit for exploring micro-and-macroviews of gases. In: Roberts N, Feurzeig W, Hunter B, editors. Computer modeling and simulation in science education. Berlin: Springer Verlag; 1999. p. 151-178.

[23] Banks J. Handbook of simulation: principles, methodology, advances, applications, and practice. Georgia: John Wiley \& Sons, Inc; 1998.

[24] Stigberg D. An introduction to the Netlogo modeling environment. In: Westervelt J D, Cohen G L, editors. Ecologist-developed spatially explicit dynamic landscape models, modeling dynamic systems. New York: Springer; 2012.

[25] World Commission on Environment and Development. Our common future. Oxford: Oxford University Press; 1987.

[26] Huerta-Barientos A, Lara-Rosano F. An initiative of Mexican Government towards sustainable rural development and innovation: The national network RENDRUS case. In: Lasker G E, Hiwaki K, editors. Sustainable development and global community. Ontario: The International Institute for Advances Studies in Systems Research and Cybernetics (IIAS); 2016.

[27] Wilkinson A, Kupers R, Mangalagiu D. How plausibility-based scenario practices are grappling with complexity to appreciate and address 21st century challenges. Technological Forecasting \& Social Change. 2013; 80: 699-710.

[28] Schoemaker P J H. Multiple scenario development: its conceptual and behavioral foundation. Strategic Management Journal. 1993; 14 (3): 193-21.

[29] Peterson G D, Cumming G S, Carpenter S R. Scenario planning: a tool for conservation in an uncertain world. Conservation Biology. 2003; 17 (2): 358-366. 
